\title{
NOVAS EMERGÊNCIAS NO TERRITÓRIO DE ALAGOAS: DIFUSÃO DA PANDEMIA COVID-19, ROMPIMENTO DE BARRAGEM E AFUNDAMENTO DE BAIRROS
}

\section{NEW EMERGENCIES IN THE ALAGOAS TERRITORY: DIFFUSION OF THE PANDEMIC COVID- 19, DAM BREAKDOWN AND NEIGHBORHOOD SINKING}

\author{
Neison Cabral Ferreira Freire \\ Doutor em Geografia \\ Fundação Joaquim Nabuco - Fundaj/Dipes/Cieg \\ neison.freire@fundaj.gov.br \\ Regina Dulce Barbosa Lins \\ Ph.D Estudos Urbanos \\ Universidade Federal de Alagoas - UFAL/FAU/NEST \\ reginalins.br@gmail.com \\ Débora Cavalcanti \\ PhD em Planejamento Urbano \\ Universidade Federal de Alagoas - UFAL/FAU/NEST \\ debora cavalcanti@hotmail.com
}

\section{RESUMO}

Esta nota técnica se refere a um estudo emergencial sobre a sobreposição e simultaneidade de três emergências no estado de Alagoas, sendo que uma delas, a pandemia da Covid-19, tem o potencial de agravar os impactos nesse território. O objetivo principal foi mapear e analisar esta simultaneidade, bem como apresentar uma primeira discussão sobre as características da vulnerabilidade social nesse cenário, bem como apresentar uma metodologia de análise espacial trabalhando com distintas escalas geográficas, a partir de dados georreferenciados dos casos confirmados da Covid-19 no período de 25 a 30 de abril de 2020. Para uma melhor condição de análise foi criado um Índice de Vulnerabilidade Social (IVS) a partir de variáveis censitárias, bem como um Coeficiente de Casos Confirmados por Distritos Sanitários para a capital, Maceió. Constatou-se, assim, o padrão espacial da difusão da pandemia no estado através dos principais eixos rodoviários, bem como a influência do polo difusor do Recife, mostrando a importância da rede de cidades e sua hierarquia urbana na difusão da pandemia. Em Maceió, observou-se que os maiores coeficientes ainda estavam nas áreas nobres, embora todos os distritos sanitários apresentassem casos confirmados já nessa data.

Palavras-chave: Covid-19. Mapas pandemia. Alagoas. Maceió.

\begin{abstract}
This technical note refers to an emergency study on the overlap and simultaneity of three emergencies in the state of Alagoas, one of which, the Covid-19 pandemic, has the potential to aggravate impacts in that territory. The main objective was to map and analyze this simultaneity, as well as to present a first discussion about the characteristics of social vulnerability in this scenario, as well as to present a spatial analysis methodology working with different geographical scales, based on georeferenced data from the confirmed cases of Covid- 19 in the period from 25 to 30 April 2020. For a better analysis condition, a Social Vulnerability Index (IVS) was created based on census variables, as well as a Coefficient of Cases Confirmed by Health Districts for the capital, Maceió. Thus, the spatial pattern of the spread of the pandemic in the state through the main roads was found, as well as the influence of the diffuser pole of Recife, showing the importance of the network of cities and their urban hierarchy in the spread of the pandemic. In Maceió, it was observed that the highest coefficients were still in the rich areas, although all health districts had confirmed cases already on that date.
\end{abstract}

Key words: Covid-19. Pandemic maps. Alagoas. Maceió.

Recebido em: 08/05/2020

Aceito para publicação em: 21/05/2020. 
Novas emergências no território de Alagoas: difusão da pandemia covid-19, rompimento de barragem e afundamento de bairros
Neison Cabral Ferreira Freire Regina Dulce Barbosa Lins Débora Cavalcanti

\section{NOTA TÉCNICA}

Este estudo emergencial, conduzido por professores e pesquisadores do Centro Integrado de Estudos Georreferenciados para a Pesquisa Social (Cieg) da Fundação Joaquim Nabuco (Fundaj) e do Núcleo de Estudos do Estatuto da Cidade (NEST) da Faculdade de Arquitetura e Urbanismo (FAU) da Universidade Federal de Alagoas (UFAL), mostram a simultaneidade de três novas emergências no território alagoano, tornando-se um caso singular entre os estados brasileiros durante a pandemia do Covid-19.

A primeira emergência que preocupa a todos em Alagoas está relacionada à expansão e conquista do território alagoano pela pandemia do Covid-19. Este estudo emergencial observou dados relativos à última semana de abril (25 a 30) e constatou, embora de forma preliminar dada a pouca capilaridade dos dados, que a pandemia por coronavírus está se instalando no território alagoano a partir do contágio por difusão, que se dá em seu epicentro, a capital Maceió.

Na última semana de abril em vídeo divulgado em várias redes sociais a diretora do Hospital Escola Dr. Hélvio Auto, Dra. Luciana Pacheco, informou que o vírus já se encontrava em mais de 40 dos 50 bairros maceioenses. $\mathrm{O}$ vírus se distribui a partir da capital, em contágio por hierarquia, pela rede de cidades e pelos corredores rodoviários, tendendo a evoluir por dois eixos em Alagoas. O primeiro deles abrange as cidades ao longo da rodovia federal BR-101 Norte, em direção a Palmares (PE), o que pode caracterizar uma expansão em duplo sentido já que o estado de Pernambuco é um difusor importante da doença. O segundo eixo de interiorização do coronavírus segue pelos corredores rodoviários alagoanos em direção à sua segunda maior cidade, Arapiraca.

Figura 1 - Mapas da Covid-19 em Alagoas - 25 (esquerda) e 30 (direita) de abril de 2020.
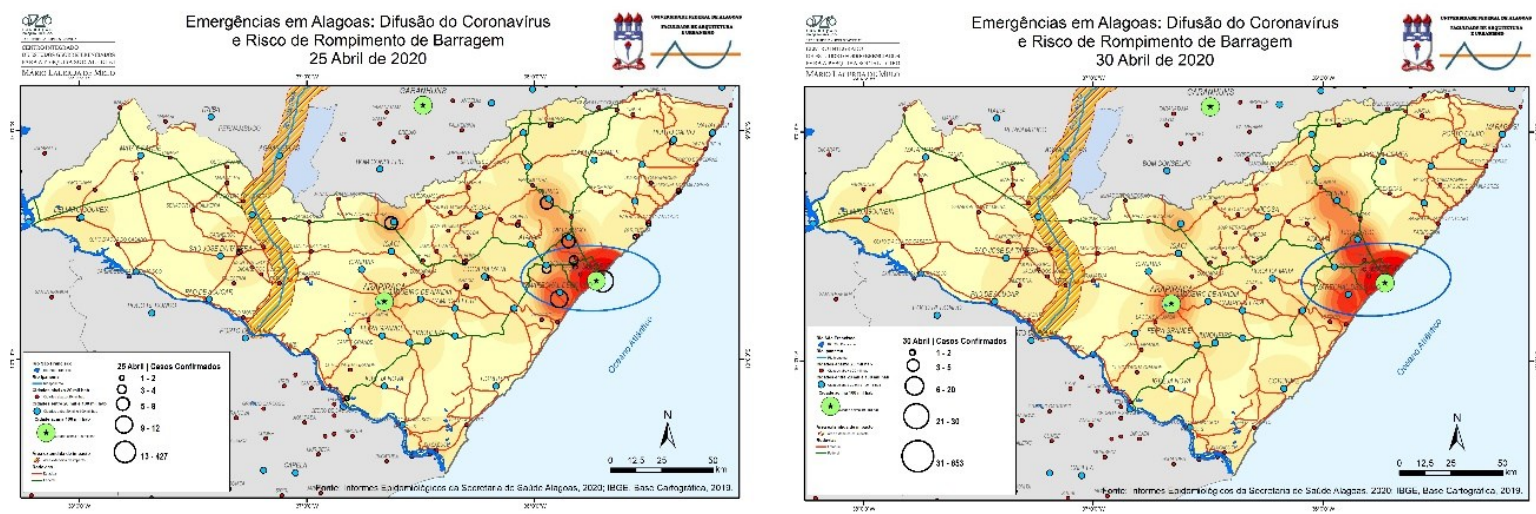

Em apenas uma semana é possível observar nos mapas o aumento da densidade de casos confirmados da doença, formando um eixo de dispersão principal do coronavírus em Alagoas na direção noroeste. Entretanto, o coeficiente de casos confirmados por 100 mil habitantes em Alagoas está sendo bem inferior ao de Pernambuco, o seu estado vizinho ao norte. Em Alagoas, no dia 30 de abril o coeficiente registrou 31 casos por 100 mil habitantes, enquanto em Pernambuco, apresentou no mesmo dia mais que o dobro, 64,7 casos por 100 mil habitantes.

Já no dia 15 de abril, frente aos números crescentes da pandemia em Pernambuco, o governo de Alagoas instalou barreiras sanitárias nas divisas com aquele estado, aplicando questionários e procedendo à medição de temperatura em todos os viajantes vindos de Pernambuco para Alagoas. Esta ação pode ter retardado a difusão do vírus no território alagoano via essas fronteiras. Contudo não é possível afirmar se esta diferença tão importante entre os coeficientes, um sendo mais do que o dobro do outro, corresponde de fato ao que acontece no território de Alagoas. Pernambuco tem produzido dados e evidências que parecem colocá-lo numa melhor situação de construção de 
narrativas sobre a doença do que a que se apresenta em Alagoas, embora a sub-notificação seja uma realidade de todos os estados brasileiros.

Para observar a pandemia em Maceió, elaborou-se um mapa com os últimos dados disponibilizados pela Secretaria de Saúde da capital, somente disponibilizados oficialmente em nível de distrito sanitário. As limitações desta análise partem da escala destes dados básicos. Não há um grau de granularidade suficiente para descrever de forma consequente, e a partir da agregação em oito distritos, o que ocorre em 50 bairros diversos de Maceió. Há que se levar em conta também a geografia peculiar desta cidade, o que pode, conforme já suscitam algumas hipóteses, afetar a forma como o vírus circula em um território. Grotas, encostas, beiras de cursos d'água, planície e tabuleiro configuram uma paisagem física recortada por desigualdades estruturais. Conscientes dessas enormes limitações, oferece-se aqui uma primeira aproximação sobre a situação do coronavírus em Maceió.

A partir do que se conhece de outras realidades urbanas brasileiras que já demonstram um avanço perverso do vírus sobre os territórios populares (aqueles mais precarizados nas cidades), construiuse, então, um Coeficiente de Contágio por 1.000 habitantes, para cada um dos oito distritos sanitários de Maceió. Optou-se não se trabalhar com números absolutos de contaminados por distrito sanitário por acreditar que outras dimensões deveriam ponderar esses números. Assim, sob esta camada foi criado um Índice de Vulnerabilidade Social, baseado em cinco variáveis do Censo 2010 (renda até 1/2 salário mínimo; presença de menores até 15 anos; precariedade de abastecimento de água; coleta de lixo e falta de esgotamento sanitário adequado).

Figura 2 - Mapa do Índice de Vulnerabilidade Social e Coeficiente de Contágio do Coronavírus em Maceió por Distrito Sanitário em 30 de abril de 2020

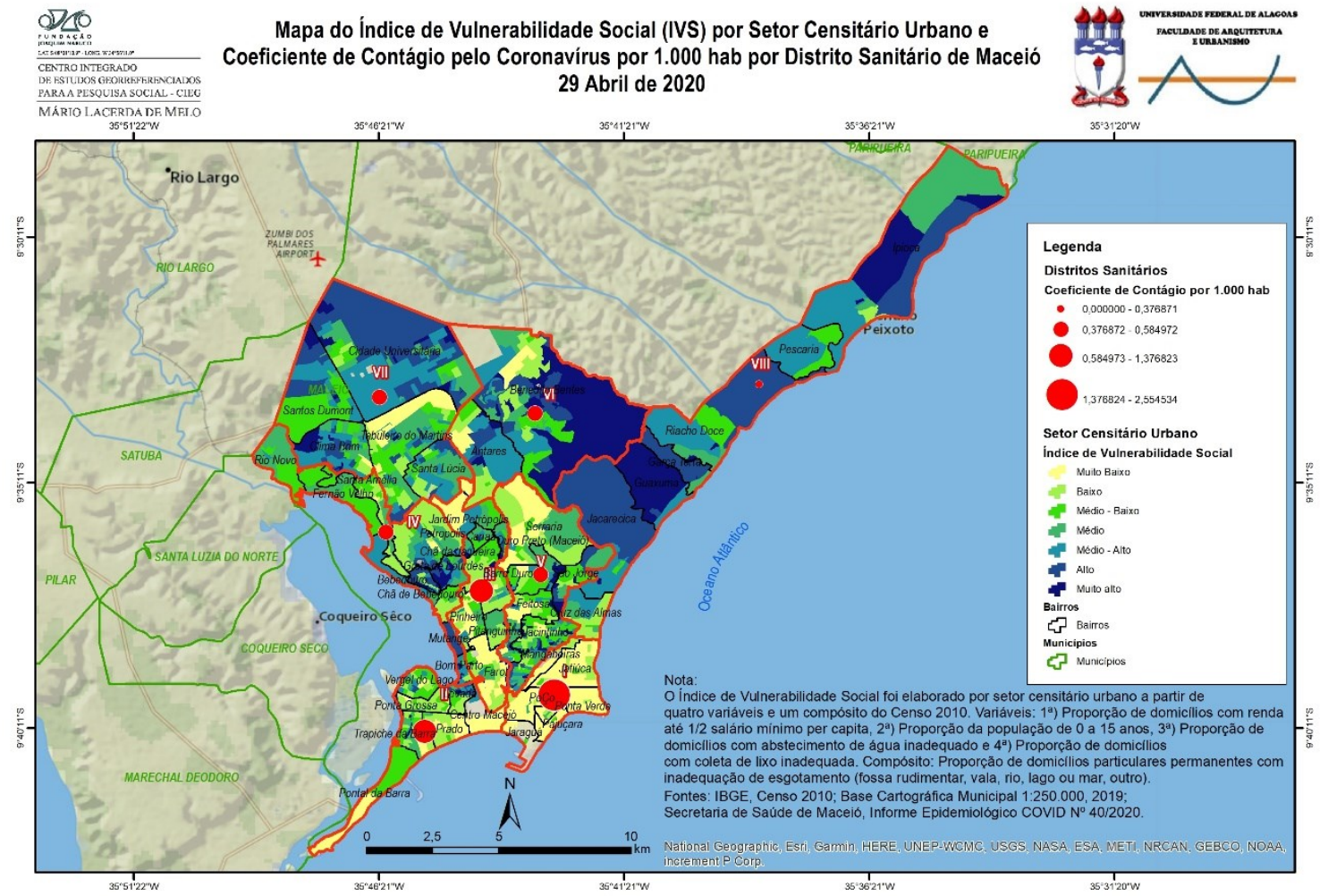

O mapa resultante demonstra a complexidade do território da cidade de Maceió entrecortado por bolsões vulneráveis em toda a sua extensão, à exceção, de forma muito genérica, de algumas porções nos entornos da orla marítima adjacente ao bairro da Ponta Verde (área nobre) e do eixo Av. 
Fernandes Lima (principal corredor de acesso da cidade). A vulnerabilidade social apresenta-se alta e concentrada na porção norte da cidade, ao longo do litoral, sobe o tabuleiro na direção noroeste assim como em porções situadas na orla da lagoa Mundaú, nas encostas que sobem pelo lado sudoeste. As grotas que recortam todo este território têm valas de drenagem na direção da lagoa Mundaú e do Oceano Atlântico e são também porções de alta vulnerabilidade.

Entretanto, e apesar das complexidades postas pela interseção da vulnerabilidade social com estruturas físicas dos assentamentos, ainda não é possível descrever a pandemia de forma mais capilarizada pela inacessibilidade aos dados por origem de residências de casos confirmados. Dessa maneira, a análise possível de ser realizada revela que a maior concentração do Covid-19, ou seja, a área de primeira magnitude, em função do índice calculado, ainda está nos bairros de situação de vulnerabilidade muito baixa, representada pela área nobre da capital. Também é possível concluir, pelo menos até esta data (30), que a pandemia (ainda) não se intensificou nas áreas de maior vulnerabilidade social da capital. Entretanto é possível perceber que o contágio por difusão já é real em todo o território maceioense, mas com menores coeficientes no seu litoral norte.

A segunda emergência em Alagoas se soma à rápida difusão da pandemia no estado: o risco de rompimento da barragem de Águas Belas (PE) com impacto em vários municípios da bacia do Rio Ipanema, situados no sertão alagoano. Cerca de 80 mil pessoas vivem em cidades às margens daquele rio em Alagoas, no seu percurso até sua foz que deságua no rio São Francisco, no município de Belo Monte. Esses municípios se caracterizam por possuírem elevadas proporções de populações com alta vulnerabilidade social, herança das desigualdades sociais e regionais que persistem na região Nordeste.

Nessas margens do Ipanema (assim como em tantos outros rios no Brasil) ocupações por habitações e construções precárias fazem parte da paisagem. Neste momento, o acúmulo dessas duas emergências, uma em curso e outra potencial, ameaçam populações com pouca ou nenhuma resiliência se o duplo desastre se soma. Considerando-se a gestão de risco, questiona-se, por exemplo, onde abrigar possíveis atingidos pelo desastre em meio a uma pandemia?

$\mathrm{O}$ risco de rompimento da barragem se torna mais ameaçador quando se tem em conta a aproximação do período chuvoso na região que, junto com a previsão de um inverno com regime pluviométrico mais intenso para este ano, poderá intensificar o risco de novas inundações. Ou seja, a drenagem de uma possível chuva intensa aumenta o risco de rompimento e a soma dos dois fatores magnifica o desastre para as cidades à jusante da barragem, podendo potencializar os danos causados.

A terceira e última emergência vincula a pandemia ao risco de um desastre tecnoindustrial na capital. Nesse sentido, Maceió representa um caso único dentre as situações urbanas que sofrem o impacto do coronavírus. Aqui, aliada a esta emergência de saúde pública e econômica, encontra-se outra emergência que se relaciona com o afundamento de porções de quatro bairros da cidade, densamente ocupados: Pinheiro, Mutange, Bebedouro e Bom Parto. Alguns desses bairros têm grandes extensões no nível da lagoa Mundaú que poderão sofrer, além do afundamento do solo como em outras áreas na parte alta, uma inundação pelo corpo hídrico lagunar. Essas áreas foram objeto por mais de 30 anos de atividades de mineração do salgema, realizadas através da perfuração de poços profundos no seu subsolo. Relatórios técnicos feito por especialistas mostraram o risco real de subsidência do solo nessas áreas, o que já motivou a remoção de 2.200 famílias até o momento.

Entretanto, os planos de emergência elaborados não foram atualizados no novo cenário da pandemia e a consequente necessidade de isolamento social. Nos planos atuais constam estratégias de mobilização da população para pontos de contato, remoção e abrigo na emergência do desastre. Então, surge a questão de como transportar e aglomerar pessoas em ginásios e escolas em meio à pandemia? Além do mais, registram-se nesses bairros áreas de alta vulnerabilidade social. Alguns habitantes ainda permanecem na área por razões distintas, mesmo ameaçados pelo possível desastre. Os bairros vêm sofrendo com o abandono das casas, aumento de arboviroses, fissuras nas construções e no solo. Essas áreas foram condenadas e se converteram em verdadeiros bairros fantasmas dentro da cidade.

Concluindo a nota, observa-se que existem heranças de desigualdades sociais em Alagoas que tornam exponenciais os impactos das novas emergências abordadas, criando enlaces e redes que se 
utilizam e se fortalecem das características do território. Estas três novas emergências em um cenário de tantas outras emergências no estado (analfabetismo, pobreza extrema, falta de oportunidades de trabalho, alta concentração de renda, dentre outras) se constituem em novos e antigos desafios para a melhoria da qualidade de vida do povo alagoano. Faz-se necessária uma agenda de políticas públicas que seja eficiente, participativa, bem formulada, correta e continuamente executada. As novas emergências, portanto, colocam em evidência o desafio urgente dessas demandas sociais. 